\title{
Blood Pool Agent CE-MRA: Improved Arterial Visualization of the Aortoiliac Vasculature in the Steady-State Using First-Pass Data
}

\author{
C.M. van Bemmel ${ }^{1 \star}$, W.J. Niessen ${ }^{1}$, O. Wink ${ }^{1}$, B. Verdonck ${ }^{2}$, and \\ M.A. Viergever ${ }^{1}$ \\ 1 Image Sciences Institute, Room E 01.334, University Medical Center Utrecht, \\ Heidelberglaan 100, 3584 CX Utrecht, the Netherlands, \\ $\{$ kees, wiro, onno, $\max \}$ @isi.uu.nl \\ 2 EasyVision Advanced Development, Philips Medical Systems, \\ Postbus 10000, Best, the Netherlands \\ bert.verdonck@philips.com
}

\begin{abstract}
Blood pool agent (BPA) contrast-enhanced magnetic resonance angiography (CE-MRA) images have been acquired during the first-pass of the contrast agent and in the steady-state. Arterial visualization, which is hampered in the steady-state owing to simultaneous enhancement of arteries and veins, can be improved if the central arterial axis (CAA) is known. A method is presented that utilizes the first-pass data to find the CAA in the steady-state data with minimum user interaction. The accuracy of the resulting CAA is compared to tracings of two observers in three datasets. It was found that the average error of the method is $0.73 \mathrm{~mm}$ in the first-pass data and $1.54 \mathrm{~mm}$ in the steady-state data.
\end{abstract}

\section{Introduction}

Blood pool agents for CE-MRA have a prolonged intravascular halflife and provide strong $\mathrm{T}_{1}$-relaxation even at low resolution [1]. Therefore, these agents allow imaging in the steady-state, thus providing longer time windows for image acquisition, which can be advantageous if high contrast and/or resolution is required. However, an important drawback is the simultaneous enhancement of arteries and veins, which hampers the interpretation of the steady-state data (see Fig. 2).

In this paper it is investigated whether information from the arteriogram acquired in the first-pass of the contrast agent can be used for improved arterial visualization of the steady-state images. Hereto a path tracking tool is utilized, which automatically outlines the central arterial axes (CAA) of the aortoiliac region in the first-pass images based on four user defined points. Subsequently, by registering the first-pass and steady-state data, the path is transferred to the steady-state. A number of visualization techniques can be utilized once the CAA is known in the steady-state images.

\footnotetext{
* This work is funded by Philips Medical Systems, Best, The Netherlands.
} 
In order to evaluate the accuracy of the method, the obtained paths are compared with manual tracings of two observers.

\section{Method}

The CAA can be used to improve arterial visualization in steady-state BPA CEMRA. Owing to the proximity of arteries and veins in the steady-state dataset, and the possible presence of pathologies, which mainly occur in the arteries, the CAA cannot readily be obtained from the steady-state data. Therefore, a method is introduced that utilizes images acquired during the first-pass of the contrast agent, in which only the arterial phase is enhanced, to estimate the CAA in the steady-state data. Hereto, first the CAA is determined in the first-pass image by finding a minimum cost path between user-defined points in an image where vessel-like structures are enhanced. Subsequently, this axis is transformed to the steady-stated data. The different steps are detailed below.

\subsection{Vesselness Filter}

A filter is employed which analyzes the local second-order image structure to determine the likeliness of a voxel to be part of a tubular structure 2]. The local image structure of an image $L$ in the neighbourhood of a point $\mathbf{x}_{0}$ can be described with the Taylor-expansion:

$$
L\left(\mathbf{x}_{0}+\delta \mathbf{x}_{0}, \sigma\right) \approx L\left(\mathbf{x}_{0}, \sigma\right)+\delta \mathbf{x}_{0}^{T} \nabla_{0, \sigma}+\delta \mathbf{x}_{0}^{T} H_{0, \sigma} \delta \mathbf{x}_{0}
$$

where $\nabla_{0, \sigma}$ and $H_{0, \sigma}$ are the gradient vector and Hessian matrix of the image computed in $\mathbf{x}_{0}$ at scale $\sigma$, respectively.

The Hessian matrix at a given voxel $\mathbf{x}$ is defined as:

$$
H(\mathbf{x}, \sigma)=\left[\begin{array}{lll}
L_{x x}(\mathbf{x}, \sigma) & L_{x y}(\mathbf{x}, \sigma) & L_{x z}(\mathbf{x}, \sigma) \\
L_{y x}(\mathbf{x}, \sigma) & L_{y y}(\mathbf{x}, \sigma) & L_{y z}(\mathbf{x}, \sigma) \\
L_{z x}(\mathbf{x}, \sigma) & L_{z y}(\mathbf{x}, \sigma) & L_{z z}(\mathbf{x}, \sigma)
\end{array}\right]
$$

where $L_{\xi_{1} \xi_{2}}(\mathbf{x}, \sigma)$ denote regularized derivatives of the image $L(\mathbf{x})$, which are obtained by convolving the image with the derivatives of the Gaussian kernel at scale $\sigma$ :

$$
\begin{gathered}
L_{\xi_{1} \xi_{2}}(\mathbf{x}, \sigma) \triangleq \sigma^{2} \frac{\partial^{2} G(\mathbf{x}, \sigma)}{\partial \xi_{1} \partial \xi_{2}} * L(\mathbf{x}) \\
G(\mathbf{x}, \sigma) \triangleq \frac{1}{\sqrt{\left(2 \pi \sigma^{2}\right)^{3}}} e^{-\frac{\|\mathbf{x}\|^{2}}{2 \sigma^{2}}}
\end{gathered}
$$

The principal directions in which the local second-order structure of the image can be decomposed are obtained by eigenvalue analysis of the Hessian matrix. Let $\lambda_{\sigma, k}$ denote the ordered eigenvalues corresponding to the $k$-th normalized eigenvector $\hat{\mathbf{u}}_{\sigma, k}$, i.e. $\left|\lambda_{\sigma, 1}\right| \leq\left|\lambda_{\sigma, 2}\right| \leq\left|\lambda_{\sigma, 3}\right|$. The eigenvectors $\hat{\mathbf{u}}_{\sigma, k}$ compose three 
orthonormal directions: $\hat{\mathbf{u}}_{1}$ indicates the direction along the vessel (minimum intensity variation), $\hat{\mathbf{u}}_{2}$, and $\hat{\mathbf{u}}_{3}$ form a base of the orthogonal plane. For an ideal tubular structure in 3D images we have:

$$
\left|\lambda_{1}\right|=0,\left|\lambda_{1}\right| \ll\left|\lambda_{2}\right|, \lambda_{2}=\lambda_{3}
$$

where the sign of $\lambda_{2}$ and $\lambda_{3}$ determines whether it concerns a bright or a dark vessel.

This approach has an intuitive justification (see Fig. 1); the second-order derivative of a Gaussian kernel at scale $\sigma$ generates a kernel that measures the contrast between the regions inside and outside the range $(-\sigma, \sigma)$. In regions with bright vessels, $\lambda_{2}$ and $\lambda_{3}$ will be large and negative.

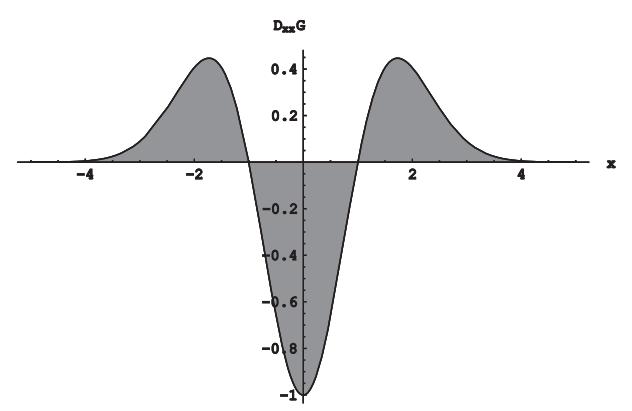

Fig. 1. Second-order derivative of a Gaussian kernel, with $\sigma=1$.

In order to estimate the likeliness of a voxel to belong to a vessel based on analysis of the eigenvalues, two geometric ratios $\left(\mathcal{R}_{A}\right.$ and $\left.\mathcal{R}_{B}\right)$, and one ratio $(\mathcal{S})$ for distinguishing background voxels from vessel voxels are introduced:

$$
\mathcal{R}_{A} \triangleq \frac{\left|\lambda_{2}\right|}{\left|\lambda_{3}\right|}, \mathcal{R}_{B} \triangleq \frac{\left|\lambda_{1}\right|}{\sqrt{\left|\lambda_{2} \lambda_{3}\right|}}, \mathcal{S} \triangleq\|\mathcal{H}\|_{F}=\sqrt{\sum_{j} \lambda_{j}^{2}}
$$

The ratio $\mathcal{R}_{A}$ is essential for distinguishing between plate-like and line-like structures. The ratio $\mathcal{R}_{B}$ accounts for the deviation from a blob-like structure. $\mathcal{S}$ is a measure of "second-order structuredness", and will be low in the background where no structure is present. In regions with high contrast compared to the background, the norm will become larger since at least one of the eigenvalues will be large.

Since in CE-MRA vessels give higher signal than the background, the output of the vessel enhancement filter at a single scale, $\sigma$, is therefore defined as:

$$
\mathcal{V}(\sigma) \triangleq \begin{cases}0 & \text { if } \lambda_{2}>0 \text { or } \lambda_{3}>0 \\ \left(1-e^{-\frac{\mathcal{R A}_{A}^{2}}{2 \alpha^{2}}}\right) e^{-\frac{\mathcal{R}_{\mathcal{B}}^{2}}{2 \beta^{2}}}\left(1-e^{-\frac{\mathcal{S}^{2}}{2 \gamma^{2}}}\right) & \text { otherwise }\end{cases}
$$


The parameters $\alpha, \beta$ and $\gamma$ tune the sensitivity of the filter to deviations in $\mathcal{R}_{A}, \mathcal{R}_{B}$ and $\mathcal{S}$ relative to the ideal behaviour for a line structure. Equation 7 explicitly states that the filter response is a function of the scale at which the Gaussian derivatives are computed. The filter is applied at multiple scales that span the range of expected vessel widths according to the imaged anatomy. In order to provide a unique filter output for each voxel, the multiple scale outputs undergo a scale selection procedure, i.e., the maximum filter response across the scales is selected:

$$
\mathcal{V}(\mathbf{x})=\max _{\sigma_{\min } \leq \sigma \leq \sigma_{\max }} \mathcal{V}(\mathbf{x}, \sigma)
$$

This way, different vessel sizes will be detected at their corresponding scales and both small and large vessels will be captured with the same scheme.

\subsection{Path Tracking}

The CAA in the first-pass data is estimated by finding the minimal cost path (MCP) in the vessel-enhanced image between user-defined points [3]. For reasons of clarity, from here on we will refer to the estimate of the CAA as the MCP. The MCP between two points is determined using a bi-directional search. In uni-directional algorithms, the search proceeds from the starting node forward until the goal node is encountered. In a bi-directional search the number of evaluations is reduced by starting a search-tree from both the starting node and the goal node simultaneously. The evolutions of the search tree are continued until the two fronts meet. The MCP is calculated using a feature image, in which the reciprocal vessel image as determined by Equation 7 represented the costs. Costs are normalized with respect to the length in order to cope with diagonal transitions and (possible) anisotropic voxels.

\subsection{Registration}

To correct for possible patient motion between acquisition of the first-pass and steady-state datasets, the first-pass dataset is rigidly registered to the steadystate dataset. Alignment is achieved by maximizing the mutual information 45. The MI registration criterion states that two images are geometrically aligned by the transformation $T^{\star}$ for which $I(u(\mathbf{x}), v(T(\mathbf{x})))$ is maximal:

$$
T^{\star}=\arg \max _{T} I(u(\mathbf{x}), v(T(\mathbf{x})))
$$

where mutual information, $I$, is defined in terms of entropy and is a measure of the variability:

$$
I(u(\mathbf{x}), v(T(\mathbf{x}))) \triangleq h(u(\mathbf{x}))+h(v(T(\mathbf{x})))-h(u(\mathbf{x}), v(T(\mathbf{x})))
$$


$h($.$) is the entropy and is defined for one variable \mathbf{x}$ as:

$$
h(\mathbf{x}) \triangleq-\int p(\mathbf{x}) \ln p(\mathbf{x}) d \mathbf{x}
$$

The obtained transformation matrix $T^{\star}$ is applied to the MCP found in the first-pass dataset to obtain an estimate of the CAA in the steady-state dataset.

\section{Experiments}

In this section the experiments to obtain the automatic MCP and the way to compare it with manually drawn paths are described.

\subsection{Image Acquisition}

For this study three datasets are examined. The images are obtained with blood pool agent MS-325 (EPIX Medical, Cambridge, Mass.). The images are obtained both during the first-pass and steady-state of the contrast. The voxel size of the reconstructed images is $0.86 \times 0.86 \times 1.50 \mathrm{~mm}^{3}$, and $0.86 \times 0.86 \times 0.95[0.90-0.98]$ $\mathrm{mm}^{3}$, respectively.

\subsection{Path Tracking}

For the automatic path tracking, the vesselness image is computed at two scale ranges, depending on the anatomical region: above the aortic bifurcation $\sigma=10.0$ - $20.0 \mathrm{~mm}$ (11 scales, equal step sizes) is used, while below the aortic bifurcation $\sigma=1.0-20.0 \mathrm{~mm}$ (20 scales, equal step sizes) is applied. The MCP is initialized at four points: at the height of the renal arteries $(\mathrm{ra})$, approximately $20 \mathrm{~mm}$ above the aortic bifurcation $(a b)$, in the left and right femoral artery (lf and $r f$, respectively). The MCP for the left (right) side of the vasculature is determined from $r a$, via $a b$, to $l f(r f)$. For the evaluation of the MCP, two observers outlined the CAA twice, both in the first-pass and the steady-state datasets using a clinical workstation (EasyVision, Philips Medical Systems) with an interval of approximately two weeks.

\subsection{Path Comparison}

To determine the distance between two paths, $\mathbf{C}_{\mathbf{1}}$ and $\mathbf{C}_{\mathbf{2}}$, first the corresponding parts are determined and resampled in $\mathrm{N}$ points. Subsequently, a symmetric mean path, $\overline{\mathbf{C}}$ is constructed by averaging all corresponding path points. For all sample points $i$ the distance $D\left(\mathbf{C}_{\mathbf{1}}, \mathbf{C}_{\mathbf{2}}, i\right)$ between the two paths is determined as:

$$
D\left(\mathbf{C}_{\mathbf{1}}, \mathbf{C}_{\mathbf{2}}, i\right)=d\left(\mathbf{C}_{\mathbf{1}}, \overline{\mathbf{C}}(i)\right)+d\left(\mathbf{C}_{\mathbf{2}}, \overline{\mathbf{C}}(i)\right)
$$

where $d\left(\mathbf{C}_{\mathbf{1}}, \overline{\mathbf{C}}(i)\right)$ is the smallest distance between the $i$-th sample point of $\overline{\mathbf{C}}$ and a densely resampled (sample rate $0.01 \mathrm{~mm}$ ) version of $\mathbf{C}_{\mathbf{1}}$. From these 
measurements the maximal distance $\left(D_{\max }\right)$, and mean distance $\left(D_{\text {mean }}\right)$ are calculated. This procedure is performed to find the mean path of each observer $\left(\overline{\mathbf{C}}_{\mathbf{1}}\right.$ and $\overline{\mathbf{C}}_{\mathbf{2}}$, respectively), as well as the mean path of the two observers $\left(\overline{\mathbf{C}}_{\overline{\mathbf{1 2}}}\right.$, the mean of $\overline{\mathbf{C}}_{\mathbf{1}}$ and $\overline{\mathbf{C}}_{\mathbf{2}}$ ). The intra-observer variability is defined as the distance of individual tracings of an observer to the observers' mean path. The interobserver variability is defined by using the averaged path, computed from all individual paths, yielding a gold standard. The gold standard is also used for testing the accuracy of the automatically determined CAA.

\section{Results}

In this section the intra-observer and inter-observer differences, and the error of the automated method are shown for the first-pass data in Table 1 and for the steady-state data in Table 2 The results are averaged over the three datasets and both legs. The average mean and maximum value and the corresponding ranges are listed.

Table 1. Mean intra-observer difference and mean inter-observer difference, and the average result of the automated method (MCP) in the first-pass data.

\begin{tabular}{lll}
\hline \hline & Mean error $(\mathrm{mm})$ & Maximum error $(\mathrm{mm})$ \\
\hline \hline Intra observer $(\mathrm{I})$ variability & $0.41[0.30-0.55]$ & $1.72[0.87-2.45]$ \\
\hline Intra observer $(\mathrm{II})$ variability & $0.34[0.31-0.40]$ & $1.31[0.95-1.53]$ \\
\hline Inter observer variability & $0.46[0.34-0.71]$ & $1.60[0.96-2.83]$ \\
\hline MCP vs $\overline{\mathbf{C}}_{\overline{\mathbf{1 2}}}$ & $0.73[0.64-0.79]$ & $2.18[1.66-3.08]$ \\
\hline \hline
\end{tabular}

Table 2. Mean intra-observer difference and mean inter-observer difference, and the average results of the automated methods, without (MCP) and with registration $\left(\mathrm{MCP}_{\text {reg }}\right)$.

\begin{tabular}{lll}
\hline \hline & Mean error $(\mathrm{mm})$ & Maximum error $(\mathrm{mm})$ \\
\hline \hline Intra observer $(\mathrm{I})$ variability & $0.43[0.35-0.51]$ & $1.89[1.40-2.73]$ \\
\hline Intra observer $(\mathrm{II})$ variability & $0.41[0.36-0.53]$ & $1.21[0.92-1.52]$ \\
\hline Inter observer variability & $0.53[0.40-0.77]$ & $1.91[0.94-3.69]$ \\
\hline $\mathrm{MCP}$ vs $\overline{\mathbf{C}}_{\overline{\mathbf{1 2}}}$ & $1.55[0.95-2.44]$ & $3.46[2.54-5.46]$ \\
\hline $\mathrm{MCP}_{\text {reg }}$ vs $\overline{\mathbf{C}}_{\overline{\mathbf{1 2}}}$ & $1.54[0.86-2.21]$ & $3.49[2.36-5.43]$ \\
\hline \hline
\end{tabular}

From the Tables it can be observed that the differences between the observers ( 0.46 and $0.53 \mathrm{~mm}$, respectively) and average differences between two tracings of 
one observer ( 0.37 and $0.42 \mathrm{~mm}$, respectively) are smaller than the in-plane resolution for both the first-pass and steady-state data. The error of the automated method is almost similar in the first-pass data $(0.73 \mathrm{~mm})$, but it was observed that when placing different initialization points, the same path is found except at the initialization points. Therefore, the automated method has a better reproducibility. The error increased in the steady-state data $(1.55 \mathrm{~mm})$. This increase is expected, since the error is composed of an error in obtaining the MCP and an error in the registration. However, obtaining the central vessel axis manually from the steady-state data is a tedious procedure. By registering the first-pass and steady-state data using maximization of mutual information, the error in the steady-state data could not significantly be reduced. In all situations, the MCP is located inside the arterial vessel.
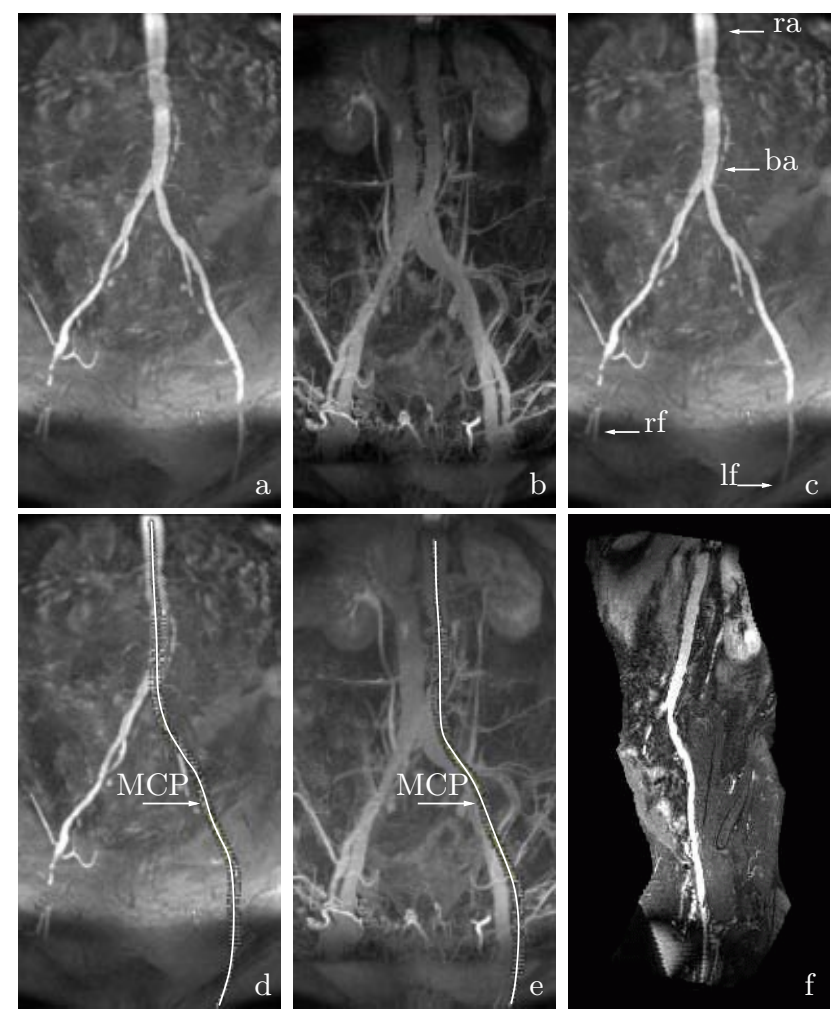

Fig. 2. Images acquired during the first-pass (a) and steady-state (b). The simultaneous enhancement of arteries and veins hampers arterial interpretation in the steady-state. After initializing four points (c, see Section 3.2) the arterial axis is estimated in first-pass data (d) and transformed, after registration to the steady-state (e). It can subsequently be used for improved arterial visualization. Here a curved reformat shows the artery over the entire segment (f), clearly displaying the path to be situated inside the artery. 
The maximum errors for the method are larger in the first-pass data and larger in the steady-state. However, closer inspection of the paths reveals that these errors occur primarily at the bifurcation, where the CAA is ill-defined. Here the observers interpolate the path differently than the automated method.

\section{Discussion}

Arterial visualization in steady-state blood pool agent CE-MRA can be improved if the CAA is known. Moreover, the CAA can be used for further analysis of the steady-state data. A method has been presented which estimates the CAA with minimum user interaction, by utilizing an arteriogram acquired during the firstpass of the contrast agent. By comparing the method with two tracings of two observers, the accuracy of the method could be assessed. The observers traced the CAA both in the first-pass data and in the steady-state data, such that the influence of (i) errors in determining the MCP in the first-pass data, and (ii) errors owing to the misregistration of first-pass and steady-state data could both be assessed. The average error of the estimated CAA is similar in the firstpass data, but reproducibility is improved. The error is larger in the steady-state data. Registration using maximization of mutual information did not improve the accuracy of the automated method, therefore, other registration methods should be considered.

Acquiring the CAA in the steady-state by a manual observer is a tedious procedure which cannot be used in clinical practice. Moreover, for improved arterial visualization and further postprocessing it is more important that the method is sufficiently accurate. In the three datasets it is checked that the estimated CAA is everywhere located within the arterial system, which is an important prerequisite, e.g., as a preprocessing step for segmentation algorithms. The evaluation carried out in this paper needs to be extended to determine whether this still holds for a larger number of datasets.

\section{References}

1. Grist TM, Korosec FR, Peters DC, Walovitch RC, Dolan RP, Bridson WE, Yucel EK, and Mistretta CA, "SteadyState and Dynamic MR Angiography with MS325: Initial Experience in Humans," Radiology, vol. 207, pp. 539-544, 1998.

2. A. F. Frangi, W. J. Niessen, K. L. Vincken, and M. A. Viergever, "Multiscale Vessel Enhancement Filtering," in Medical Image Conference and Computer Assisted Interventions. 1998, pp. 130-137, SpringerVerlag.

3. O. Wink, W. J. Niessen, and M. A. Viergever, "Minimum Cost Path Determination Using a Simple Heuristic Function," in Proc. International Conference on Pattern Recognition - 4, September 2000, pp. 1010-1013.

4. P. Viola and W.M. Wells, "Alignment by Maximization of Mutual Information," Proc. 5th Int. Conf. on Computer Vision, pp. 26-23, June 1995.

5. F. Maes, A. Collignon, D. Vandermeulen, G. Marchal, and P. Suetens, "Multimodality Image Registration by Maximization of Mutual Information," IEEE Transactions on Medical Imaging, vol. 16, pp. 187-198, 1997. 